\title{
INCREASED CARDIAC OUTPUT CAUSED BY SECONDARY HYPERNEPHROMA
}

\author{
BY \\ GEOFFREY BOURNE \\ From St. Bartholomew's Hospital, London
}

The local vascularity occurring in some cases of Paget's disease is sufficient to establish what amounts to an arterio-venous shunt that is large enough to lower the diastolic blood pressure and to increase the left ventricular output (Edholm et al., 1945, and McMichael, 1947). The present case is that of a man, aged 72, in whom a secondary hypernephroma in the left tibia produced similar conditions.

When first examined his chief complaints were increased shortness of breath on exertion, cough, lassitude, and pain in the left shin. He had also considerable orthopnœa and was sleeping badly. He gave a history of having had a nephrectomy six years previously for hypernephroma. On examination he was very short of breath undressing, and was orthopnœic. The heart rate was 100 , the rhythm was regular. There was a large pulse pressure $(180 / 80)$. The heart sounds were normal. The electrocardiogram showed right bundle-branch block. There were signs of collapse and of impaired respiratory function over the right lower lobe. Pitting œdema was present over both lower legs. The hæmoglobin was 102 per cent.

The left tibia in its upper quarter was the site of a large, fairly firm, warm swelling (Fig. 1) measuring 7.5 by $5.0 \mathrm{~cm}$. Over the whole of this swelling expansile pulsation was felt, and a prolonged musical systolic murmur was heard. X-ray screening of the chest showed a large mass in the right lower quadrant almost certainly caused by a secondary deposit. There appeared to be a small quantity of fluid in the right pleural cavity. The radiological report was as follows: in the right lung, there was a massive opacity projecting out from the region of the right hilum, and some dense punctate shadows in the lower zone with right basal pleural effusion; the left lung was clear except for a few scars.

There was extensive bone erosion in the upper third of the left tibia anteriorly, with some swelling of the soft parts. Some alteration of the trabecular structure nearby indicated associated Paget's disease: these latter changes were slight and local, those due to the secondary deposits greatly predominating. In the upper third of the right tibia there were poorly demarcated areas of erosion and a more sharply demarcated area of bone erosion in the lower third with some cortical bone expansion.

He was thought to have a neoplasm in the region of the right hilum with pleural effusion. The tibial bone lesions were thought to be secondary deposits. There was no evidence of secondary deposits or of Paget's disease in the dorsal or lumbar spine, in the pelvis, or in the long bones.

When massive vascular secondary deposits occur in the sternum or ribs the presence over them of a systolic thrill and a systolic murmur, and the fact that they may manifest expansile pulsation, can cause them to mimic very closely aneurysms of the aorta (Dr. R. Bodley Scott, personal communication).

The patient was admitted to St. Bartholomew's Hospital for further investigation and for deep X-ray treatment of the secondary deposit in his left tibia, which was causing him much pain and disability. The investigations that are the subject of this communication were concerned with the secondary tumour in the left tibia. A phonocardiogram (Fig. 2) taken by Dr. B. G. Wells showed that the murmur in the tibia was continuous, and that it had the characteristics of the murmur of an arterio-venous fistula, being maximal at the time of the second heart sound.

Peripheral Blood Flow. Investigations on the peripheral blood flow were performed by Dr. Robert Duff. Under standard conditions (room temperature $23.0^{\circ} \mathrm{C}$; ; plethysmograph temperature $35.0^{\circ}$ C.) blood flow was measured in the feet

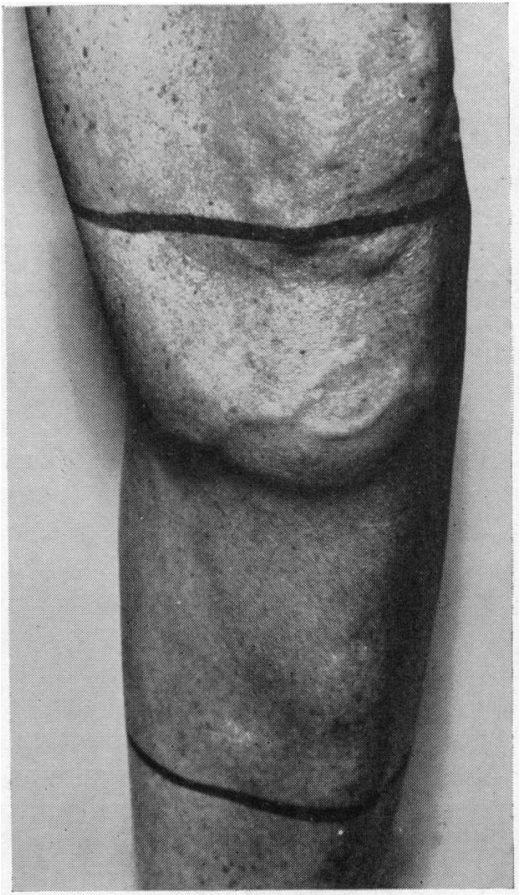

FIG. 1.-The swelling caused by the secondary hypernephroma in the upper part of the left tibia. The dark lines are guides for deep X-ray therapy. 
by venous occlusion plethysmography. The blood flow in both feet ranged between 8 and $10 \mathrm{ml} . / 100 \mathrm{ml}$. of foot volume/min., there being no significant difference between the two sides. It was possible to measure the blood flow under similar conditions in the left knee, a plethysmograph being placed so as to enclose the knee joint and patella and the upper end of the tibia including the metastatic swelling. The flow through these ranged from 15.0 to $27.0 \mathrm{ml} . / 100 \mathrm{ml}$. tissue volume $/ \mathrm{min}$. with an average of $21 \mathrm{ml}$.

The flow in the feet was high, though not outside the upper range of normal, but was not significantly greater in the left foot. In the left knee, a very high value was recorded; although comparable measurements in healthy subjects are not available there is no doubt that this represents a pathological increase, for the blood flow in the knee should be no greater than that in the foot, under these standard conditions.

Left Femoral Artery Occlusion by Digital Pressure. The diastolic pressure during the control period ranged from 70 to 76 with a mean value of $74 \mathrm{~mm}$. Hg. On occluding the left femoral artery it rose immediately to $80 \mathrm{~mm}$., then fell to 76 , and rose once more to 78 at which point it was maintained during the minute that the artery was occluded. On release of the femoral artery, the diastolic blood pressure fell after a few seconds to $74 \mathrm{~mm}$. at which level it was maintained steadily to the completion of the observation. The rise in diastolic blood pressure, though small, was constant and presumably signified that a large volume of blood was shunting from the arterial to the venous circulations in the left lower extremity. Digital occlusion of the left femoral artery was attended by an immediate fall in the heart rate from 98 to 92 a minute-further evidence that the shunt in the left lower extremity was considerable.

Finally, an angiogram on the local vascular condition by Mr. G. W. Taylor showed the presence of sizeable arterial circulation round the upper end of the left tibia.

When the patient was re-examined three months after deep X-ray treatment the shin swelling was much reduced in size, the systolic thrill and murmur had disappeared, and the pulse pressure was no longer increased (140/70).

Conclusion. It is clear from these observations that a massive vascular secondary deposit in bone can produce changes in the circulation similar to those caused by the vascular stages of Paget's disease.

\section{References}

Edholm, O. G., Howarth, S., and McMichael, J. (1945). Clin. Sci., 5, 249.

McMichael, J. (1947). Advances in Internal Medicine, 2, 64. 